\title{
Trombosis inusuales y trombofilia Un problema difícil de abordar. Experiencia de cuatro años
}

\author{
Unusual thrombosis and thrombophilia \\ A difficult problem to approach. Experience of four years
}

\author{
luis Eduardo Buitrago, Claudia Patricia Casas, María Helena Solano \\ - Bogotá, D.C. (Colombia)
}

\section{Resumen}

Existen territorios vasculares denominados sitios inusuales que pueden presentar eventos trombóticos: senos venosos, miembros superiores y lecho esplácnico, con consecuencias clínicas que pueden ser catastróficas. Se encuentran vacíos de conocimiento en el estudio de los factores involucrados en estas entidades.

Objetivo: escribir la frecuencia de trombofilia en pacientes que consultan por trombosis en sitios inusuales al servicio de hematología del Hospital San José, entre enero 2007 y diciembre 2011. Se describe la población en términos demográficos, género, edad, recurrencia, tratamientos empleados y complicaciones asociadas.

Métodos: es un estudio descriptivo serie de casos, se incluyeron las historias clínicas de pacientes mayores de 18 años con primer evento trombótico que compromete trayecto vascular en sitio inusual, asistentes a la consulta externa de hematología durante el periodo de estudio, los datos fueron extraídos de las historias clínicas de forma retrospectiva.

Resultados: en el estudio ingresaron 73 pacientes, mayoría mujeres (65.8\%), la localización de la trombosis fue: senos venosos (47.9\%), miembro superior $(27.4 \%)$, lecho esplácnico (24\%). Los factores de riesgo de mayor frecuencia fueron: embarazo (12.5\%), consumo de anovulatorios orales (10.4\%), tabaquismo (9.6\%), puerperio (4.2\%). Se encontró trombofilia en $57 \%$ de los pacientes con trombosis en sitios inusuales, siendo la más frecuente el síndrome antifosfolípidos.

Conclusiones: existe una frecuencia alta para trombofilia en pacientes con trombosis en sitio inusual, siendo el síndrome antifosfolípidos la causa más frecuente en nuestra serie. (Acta Med Colomb 2013; 38: 132-137).

Palabras clave: trombosis miembros superiores, trombosis de senos venosos, venas mesentéricas, trombofilia.

\footnotetext{
Abstract

There are vascular territories called unusual sites which may present thrombotic events: venous sinuses, upper limbs and splanchnic bed, with clinical consequences that can be catastrophic. Knowledge gaps are found in the study of the factors involved in these entities.

Objective: To describe the frequency of thrombophilia in patients who consult to the Hematology Service of San Jose Hospital due to thrombosis in unusual sites between January 2007 and December 2011. We describe the population in terms of demographics, gender, age, recurrence, treatments used and associated complications.

Methods: A case series descriptive study. We included the medical records of patients older than 18 years with first thrombotic event compromising a vascular unusual site, attending the outpatient hematology service during the study period. Data were extracted retrospectively from medical records.

Results: 73 patients entered the study; most were women (65.8\%). Localization of thrombosis was: venous sinuses (47.9\%), upper limb (27.4\%), splanchnic bed (24.0\%). The most frequent risk factors were: pregnancy $(12.5 \%)$, use of oral contraceptives (10.4\%), smoking $(9.6 \%)$, postpartum (4.2\%). Thrombophilia was found in $57 \%$ of patients with thrombosis in unusual sites. The most frequent diagnosis was antiphospholipid syndrome.
}

Dr. Luis Eduardo Buitrago: Residente II de Hematología. Fundación Universitaria de Ciencias de la Salud; Dra. Claudia Patricia Casas: Fundación Universitaria de Ciencias de la Salud, Hospital San José; Dra. María Helena Solano: Profesora Titular, Jefe Servicio Hematología Hospital San José, Fundación Universitaria de Ciencias de la Salud. Bogotá, D.C. (Colombia) Correspondencia. Dr. Luis Eduardo Buitrago. Bogotá, D.C. (Colombia) E-mail: luiseduardobn46@gmail.com Recibido: 26/X/012 Aceptado: 27/VI/2013 
Conclusions: There is a high frequency of thrombophilia in patients with thrombosis in unusual site, being antiphospholipid syndrome the most common cause in our series. (Acta Med Colomb 2013; 38: 132-137).

Keywords: upper limb thrombosis, venous sinus thrombosis, mesenteric veins, thrombophilia.

\section{Introducción}

La trombosis venosa constituye un síndrome clínico heterogéneo, con varios factores genéticos y adquiridos involucrados, siendo la trombosis en miembro inferior y territorio pulmonar los segmentos mayormente comprometidos con tasas de mortalidad y morbilidad ampliamente informados en la literatura (1-3); sin embargo, se pueden comprometer territorios vasculares denominados sitios inusuales: senos venosos, miembros superiores y lecho esplácnico este último incluye trombosis de la vena porta, trombosis de vena hepática (síndrome Budd Chiari) y trombosis mesentérica. Existen vacíos de conocimiento en el estudio de los factores involucrados en estas entidades, reportando una incidencia anual para miembro superior de dos casos por 100000 personas y corresponde $5 \%$ de todas las trombosis (4-6), para senos venosos cinco casos por millón año $(5,7,8)$. Respecto a recurrencia se reporta en cinco por 100 pacientes por año para trombosis de senos venosos (7); esta recurrencia es similar a la reportada para trombosis venosa profunda de miembros inferiores, según el trabajo Gosk con datos recolectados en Clínica Mayo entre 1978 y 2011 con una mortalidad de 2.8 por 100 pacientes año (9). A pesar de tener una incidencia baja cada evento puede llevar a compromiso importante sobre órganos vitales tales como sistema nervioso central y lecho esplácnico, con tasas de mortalidad que pueden variar entre 5 y $30 \%$ dependiendo de las comorbilidades asociadas y severidad del evento $(5,7,10)$. Se considera controversial el estudio de trombofilia en trombosis de sitios inusuales, algunos trabajos locales evidencian asociación de trombosis venosa profunda y trombofilia en la población colombiana (11), para el estudio de sitios inusuales se recurre a series internacionales que muestran asociación con trombofilia heredada en $34 \%$, en una serie de 75 pacientes con trombosis venosa de miembros superiores; en el estudio de Martinelli que incluye 115 pacientes se reporta un OR de 6.2 (IC 95\% 2.5-15.7) para mutación del Factor V de Leiden, OR 5.0 (95\% IC 2.0-12.2), para mutación del gen de la protrombina G20210, OR 4.9 (IC 95\% 1.-22.0) con una incidencia anual de recurrencia de $4.4 \%$ en paciente con trombofilia y $1.6 \%$ en pacientes sin trombofilia con un tiempo medio de anticoagulación de seis meses (4). Se ha reportado asociación entre trombosis de senos venosos y trombofilia, con especial relevancia en presencia de la mutación del Factor V Leiden, que incrementa en cinco veces el riesgo de presentar un evento trombótico, la mutación para la protrombina G20210 aumenta en 10 veces el riesgo y la hiperhomocisteinemia cuatro veces $(7,12)$. Es interesante resaltar la asociación entre trombosis venosa cerebral, trombofilia heredada y consumo de anticonceptivos orales, sinergismo documentado en un metaanálisis reciente (13). Algunas organizaciones han intentado dar algunas directrices para el tratamiento de este tipo de entidad como el grupo SISET (Sociedad Italiana de Trombosis y Hemostasia) donde propone un tiempo mínimo de anticoagulación de tres a seis meses (14).

Los diferentes prestadores de servicios de salud que se enfrentan a esta situación clínica deben tener claridad sobre las distintas pruebas diagnósticas y sus valores de referencia según los estándares propuestos para determinar una trombofilia subyacente o un estado procoagulante persistente inherente o adquirido que pueda conllevar a cambios en la estrategia terapéutica, así como el tiempo óptimo de tratamiento y el riesgo de nuevos eventos. Los estudios observacionales no han podido establecer una fuerte asociación entre fenómenos de trombofilia y sitios inusuales; algunas series han reportado mayor riesgo de eventos trombóticos respecto a la población general, siendo las conductas actuales tomadas a partir de concepto de expertos (15-22).

El objetivo principal de este trabajo fue describir la frecuencia de trombofilia en pacientes que consultaron por trombosis en sitios inusuales al servicio de hematología del Hospital San José, entre enero 2007 y diciembre 2011. Se describió la población en términos demográficos, género, edad, con una descripción de variables de recurrencia, tratamientos empleados y complicaciones asociadas, adicionalmente se evaluará si las pruebas diagnósticas cumplen con los lineamientos internacionales para el diagnóstico de trombofilia (23-25). Se espera que la información suministrada facilite el ejercicio clínico del personal que se enfrenta al manejo de pacientes con trombosis en sitios inusuales.

\section{Métodos}

Es un estudio descriptivo serie de casos, se incluyeron pacientes mayores de 18 años con primer evento trombótico que compromete trayecto vascular que involucre miembros superiores, vena mesentérica, porta, extrahepática o trombosis de senos venosos, que asistieron a la consulta externa de hematología durante el periodo comprendido entre enero de 2007 y diciembre 2011, la recolección de datos se realizó de forma retrospectiva. Se excluyeron pacientes con diagnóstico de trombosis de miembros superiores asociadas a dispositivos intravasculares. La búsqueda de las historias clínicas se realizó de la siguiente manera: inicialmente, se seleccionaron las historias clínicas con códigos CIE 10 relacionados con el diagnóstico de trombosis (I80.2, I82.7, 
D73.5, I74.8, G0.8), que corresponden a los diagnósticos de trombosis venosa profunda, trombosis esplénica y trombosis de senos venosos, de los cuales se obtuvieron 1100 historias clínicas. Posteriormente se confrontaron estas historias clínicas con el archivo electrónico del servicio de hematología, seleccionando los pacientes que cumplían los criterios de inclusión. Por último se implementó el instrumento de recolección de información, se realizó revisión manual de la historia clínica, para obtener la información allí consignada. Las historias clínicas, 73 en total, fueron suministradas por el servicio de archivo del Hospital San José (Flujograma 1). Los datos recolectados se agruparon en cinco categorías: 1) Caracterización de los pacientes, que comprende: edad, sexo, sitio comprometido, presencia de trombofilia, forma en que se realizó el diagnóstico. 2) Pruebas diagnósticas solicitadas para determinar trombofilia. 3) Factores de riesgo asociados con trombosis. 4) Tipo de tratamiento, recurrencia y suspensión de la terapia. 5) Descripción de cumplimento de estándares en el diagnóstico de trombofilia. El almacenamiento de los datos se realizó en la hoja electrónica de Excel. Con verificación por un segundo digitador para evitar errores en el paso de la información. El análisis de la información se realizó utilizando el programa estadístico Stata $12^{\circledR}$. Se utilizó estadística descriptiva. Las variables cualitativas se presentaron como frecuencias absolutas y relativas. Las variables cuantitativas se analizaron con medidas de tendencia central y dispersión. El protocolo fue aprobado por el comité de ética en investigación con seres humanos de la Fundación Universitaria de Ciencias de la Salud y se consideró un estudio sin riesgo de acuerdo con la resolución 8430 de la reglamentación colombiana.

\section{Resultados}

En el estudio se analizaron datos de 73 pacientes con edad media de 40 años (DE 14.2 años), siendo la mayoría mujeres (65.8\%). El diagnóstico del evento se realizó en

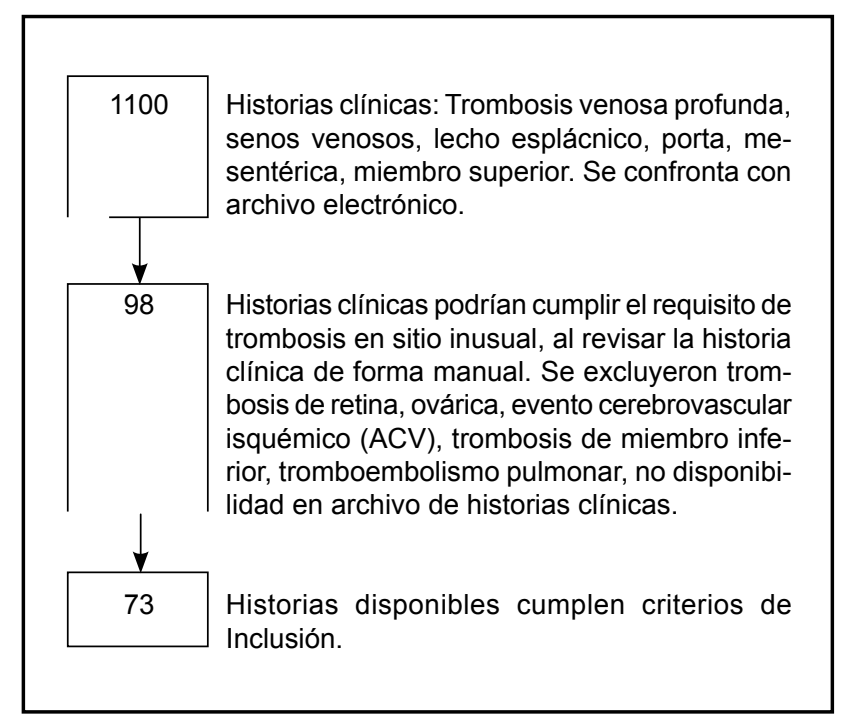

Flujograma 1. Selección de los pacientes. gran proporción de los pacientes de forma clínica (96\%), con una localización de la trombosis en senos venosos (47.9\%), miembro superior $(27.4 \%)$, lecho esplácnico $(24 \%)$. Los factores de riesgo de mayor frecuencia embarazo (12.5\%), consumo de anovulatorios orales (10.4\%), tabaquismo (9.6\%), neoplasia asociada (5.5\%), puerperio (4.2\%) (Tabla 1). Se encontró trombofilia en más de la mitad de los pacientes con trombosis en sitios inusuales (57\%), siendo la más frecuente el síndrome antifosfolípidos en 27 casos, con prueba de anticoagulante lúpico positivo en 20 pacientes, los pacientes con diagnóstico de síndrome antifosfolípidos podían tener más de una prueba positiva (anticoagulante lúpico, anticardiolipinas $\operatorname{IgG}, \operatorname{IgM}$, B2GI) (Tabla 2).

El tratamiento con warfarina fue la terapia más frecuente (74\%), seguido por la heparina de bajo peso molecular (21\%). Se encontró tiempo de tratamiento mayor de 12 meses $(85 \%)$, se suspendió la terapia por criterio médico en los pacientes con trombofilias menores o sin trombofilia (26\%) (Tabla 3).

De los 42 pacientes con trombofilia, se encontraron 25 pacientes (60\%) con compromiso de senos venosos, miembros superiores ocho pacientes (19\%), mesentérica cinco pacientes (12\%), vena porta cuatro pacientes (9.5\%), siendo el síndrome antifosfolípidos la trombofilia más frecuente en todos los grupos. Para miembros superiores se encontró en dos pacientes el déficit de proteína $S$, en tercer lugar hiperhomocisteinemia y mutación para MTHFR un paciente con cada alteración, en senos venosos la segunda

Tabla1. Características de la población n 73 .

\begin{tabular}{|c|c|c|}
\hline $\begin{array}{l}\text { Edad, años promedio (DE) } \\
\text { menores de } 40 \text { años, n (\%) }\end{array}$ & $\begin{array}{l}40 \\
39\end{array}$ & $\begin{array}{l}(14.2) \\
(53.4)\end{array}$ \\
\hline Sexo femenino, n (\%) & 48 & $(65.8)$ \\
\hline \multicolumn{3}{|l|}{ Procedencia } \\
\hline Urbano & 72 & (98.6) \\
\hline Rural & 1 & $(1.4)$ \\
\hline \multicolumn{3}{|l|}{ Diagnóstico } \\
\hline Clínico* & 70 & $(95.9)$ \\
\hline Quirúrgico.† & 3 & (4.1) \\
\hline \multicolumn{3}{|l|}{ Localización|| } \\
\hline Senos venosos & 35 & $(47.9)$ \\
\hline Lecho esplácnico $\ddagger$ & 18 & $(24.6)$ \\
\hline Porta & 7 & $(9.6)$ \\
\hline Mesentérica & 11 & (15.1) \\
\hline Extrahepática & 0 & 0 \\
\hline Miembro superior & 20 & $(27.4)$ \\
\hline \multicolumn{3}{|l|}{ Factores de Riesgo } \\
\hline Embarazo II & $6 / 48$ & (12.5) \\
\hline Puerperio & $2 / 48$ & $(4.2)$ \\
\hline Toma de AO. $\S$ & $5 / 48$ & $(10.4)$ \\
\hline Neoplasia & 4 & (5.5) \\
\hline Tabaquismo & 7 & (9.6) \\
\hline \multicolumn{3}{|c|}{$\begin{array}{l}\text { * Diagnóstico clínico apoyado con imágenes diagnósticas } \\
\text { † En intraoperatorio o en pieza de patología compatible con trombosis } \\
\text { † El lecho esplácnico se divide en porta, extrahepática, mesentérica, se describe el } \\
\text { territorio comprometido. }\end{array}$} \\
\hline
\end{tabular}


Tabla 2. Resultados frecuencia de trombofilia en pacientes con trombosis en sitios inusuales.

\begin{tabular}{|c|c|c|}
\hline Trombofilia n/número de pruebas realizadas (\%) & 42 & $(57.5)$ \\
\hline \multicolumn{3}{|l|}{ Mutaciones } \\
\hline \multicolumn{3}{|l|}{ THFR } \\
\hline Homocigoto $\neq$ & $1 / 4$ & $(25)$ \\
\hline Heterocigoto & $3 / 4$ & $(75)$ \\
\hline \multicolumn{3}{|l|}{ Factor V de Leiden } \\
\hline Heterocigoto & $1 / 26$ & (3.8) \\
\hline Homocigoto & $0 / 26$ & \\
\hline \multicolumn{3}{|l|}{ Protrombina } \\
\hline Heterocigoto & $2 / 22$ & (9) \\
\hline Homocigoto & $0 / 22$ & \\
\hline Síndrome anticuerpos antifosfolípidos. * & $27 / 65$ & $(41.5)$ \\
\hline Anticardiolipinas IgG & $7 / 54$ & (13) \\
\hline Anticardiolipinas IgM & $8 / 62$ & (13) \\
\hline Anticoagulante lúpico & $20 / 60$ & (33) \\
\hline B2GI IgM & $1 / 20$ & (5) \\
\hline B2GI IgG & $3 / 20$ & (15) \\
\hline Déficit de proteína C & $1 / 31$ & $(3.2)$ \\
\hline Déficit de proteína $S$ & $5 / 30$ & $(16.6)$ \\
\hline Déficit de antitrombina III & $3 / 43$ & (7) \\
\hline Factor VIII > 200\% & $2 / 18$ & (11) \\
\hline Hiperhomocisteinemia & $4 / 28$ & $(14.2)$ \\
\hline \multicolumn{3}{|l|}{ Cumplimiento con estándar } \\
\hline Anticoagulante lúpico† & $10 / 20$ & $(50)$ \\
\hline \multicolumn{3}{|c|}{$\begin{array}{l}\text { El númerador corresponde al número de pruebas positivas y denominador corresponde } \\
\text { al número de pruebas realizadas. }\end{array}$} \\
\hline \multirow{2}{*}{\multicolumn{3}{|c|}{$\begin{array}{l}\text { * Los pacientes con síndrome antifosfolípidos podían tener más de una prueba positiva } \\
\ddagger \text { se describieron las frecuencias dependiendo del número de pruebas realizadas a los } \\
\text { pacientes, no a todos se les realizaron todos los laboratorios }\end{array}$}} \\
\hline & & \\
\hline \multicolumn{3}{|c|}{$\begin{array}{l}\dagger \text { confirmado mediante veneno de víbora de Russell, tiempo parcial de tromboplastina } \\
\text { activado con resultado positivo }\end{array}$} \\
\hline
\end{tabular}

trombofilia más frecuente fue la mutación para MTHFR con tres pacientes, dos mutaciones heterocigotas, una mutación homocigota, le sigue en frecuencia mutación para el gen de la protrombina en dos pacientes con mutaciones heterocigotas, dos pacientes con déficit de proteína S, antitrombina III; en el lecho esplácnico en segundo lugar se encontró hiperhomocisteinemia en dos pacientes, también se encontró niveles elevados de Factor VIII, déficit de antitrombina, proteína S y $\mathrm{C}$ cada uno con un paciente (Tabla 4). En algunas series se ha reportado el sinergismo entre trombofilia y factores de riesgo provocados, en nuestra serie identificamos tres pacientes con trombosis de senos venosos de los cuales dos tenían diagnóstico de síndrome antifosfolípidos y uno mutación para gen de la protrombina, el factor de riesgo asociado fue consumo de anovulatorios orales.

\section{Discusión}

La trombosis en sitio inusual sigue siendo un problema clínico de difícil abordaje, tiene implicaciones clínicas muy importantes con tasas de morbilidad y mortalidad, nuestra serie reunió 73 historias clínicas de pacientes con trombosis
Tabla 3. Resultados de tratamiento, recurrencias, duración.

\begin{tabular}{|lcc|}
\hline Tratamiento n(\%) & & \\
Warfarina & 54 & $(74)$ \\
Heparina de bajo peso molecular & 15 & $(21)$ \\
Fondaparinaux & 2 & $(2.7)$ \\
Sin tratamiento & 2 & $2.7)$ \\
\hline Tiempo de tratamiento & & \\
$\quad$ Más de 12 meses & 62 & $(85)$ \\
De cero a tres meses & 5 & $(6.9)$ \\
De seis a 12 meses & 3 & $(4.1)$ \\
\hline Recurrencia & 3 & $(4.1)$ \\
\hline Sitio de recurrencia & & \\
Esplácnico & 1 & $(1.4)$ \\
Miembro superior & 2 & $(2.7)$ \\
$\quad$ Otros* & 1 & $(1.4)$ \\
\hline Se suspende tratamiento & 23 & $(31)$ \\
$\quad$ Criterio médico & 19 & $(26)$ \\
Sangrado mayor & 4 & $(5.5)$ \\
\hline * miembros inferiores, territorio pulmonar. & & \\
\hline
\end{tabular}

Tabla 4. Distribución de trombofilias de acuerdo a localización de trombosis.

\begin{tabular}{|c|c|c|c|}
\hline \multirow[t]{2}{*}{ Trombofilia } & $\begin{array}{c}\text { T. senos } \\
\text { venosos } \\
25(60 \%)\end{array}$ & $\begin{array}{c}\text { T. miembro } \\
\text { superior } \\
8(19 \%)\end{array}$ & $\begin{array}{c}\text { T. lecho esplácnico. } \\
\text {-Mesentérica } 5(12 \%) \\
\text {-Porta } 4(9,5 \%)\end{array}$ \\
\hline & $\mathbf{N}$ & $\mathbf{n}$ & $\mathbf{N}$ \\
\hline Mutación MTHFR & 3 & 1 & 0 \\
\hline Mutación gen de la protrombina & 2 & 0 & 0 \\
\hline Mutación Factor V Leiden & 1 & 0 & 0 \\
\hline Déficit proteína $S$ & 2 & 2 & 1 \\
\hline Déficit proteína C & 0 & 0 & 1 \\
\hline Hiperhomocisteinemia & 0 & 1 & 2 \\
\hline Déficit Antitrombina III & 2 & 0 & 1 \\
\hline Niveles Factor VIII elevados & 0 & 0 & 1 \\
\hline Síndrome antifosfolípidos (SAF) & 18 & 4 & 5 \\
\hline \multicolumn{4}{|c|}{$\begin{array}{l}\text { Comentario: se encuentran cinco pacientes con más de una prueba positiva que sugiere } \\
\text { trombofilia combinada, así:. }\end{array}$} \\
\hline \multicolumn{4}{|c|}{ Paciente 1: homocisteína - SAF. Localización senos venosos } \\
\hline \multicolumn{4}{|c|}{ Paciente 2: Antitrombina III - SAF. Localización senos venosos } \\
\hline \multicolumn{4}{|c|}{ Paciente 3: Antitrombina III - Homocisteína - SAF. Localización porta } \\
\hline \multicolumn{4}{|c|}{ Paciente 4: Factor VIII - SAF. Localización porta } \\
\hline \multicolumn{4}{|c|}{ Paciente 5: Proteína S - SAF. Localización senos venosos. } \\
\hline
\end{tabular}

en sitios inusuales, se resaltan los factores de riesgo relacionados con el sexo femenino como embarazo, puerperio y consumo de anovulatorios orales, las publicaciones internacionales reportan un tasa de 34.2 por 100000 embarazos $(26,27)$, en nuestra serie se identificó como factor de riesgo en las mujeres que presentaron trombosis en sitio inusual 
embarazo en $(12.5 \%)$, puerperio $(4.2 \%)$, consumo de anovulatorios orales $(10.4 \%)$, la frecuencia de trombofilia es variable en las diferentes series publicadas. En nuestra serie la frecuencia de trombofilia fue de $57 \%$ en los pacientes con trombosis en sitios inusuales, siendo el síndrome antifosfolípidos la trombofilia más frecuente en todos los grupos. $\mathrm{Al}$ realizar el análisis por territorio comprometido en los casos en que se pudo identificar trombofilia, para el sistema porta se identificaron cuatro pacientes $(9.5 \%)$, mesentérica en cinco pacientes (12\%), mientras que la serie de De Stefano $\mathrm{V}$ analiza trombosis en lecho esplácnico reporta trombofilia heredada en un $20-50 \%$, con una prevalencia del $30-50 \%$ para neoplasia mieloproliferativa crónica mutación JAK 2 v617f $(18,28,29)$, siendo este último uno de los factores de riesgo más importantes en la trombosis de lecho esplácnico; en nuestra serie no tenemos registros sobre esta variable ya que no fue incluida en el trabajo dado las dificultades para la realización de el examen JAK2 en su momento.

Se encontraron 25 pacientes $(60 \%)$ con trombofilia para trombosis de senos venosos, de los cuales en tres casos se encontró sinergismo entre trombofilia y factor de riesgo provocado, se identificaron dos casos con síndrome antifosfolípidos y una mutación para gen de la protrombina siendo el consumo de anovulatorios orales el factor de riesgo asociado, datos acordes al alto riesgo de trombosis descrito para la asociación entre trombosis de senos venosos, trombofilia y factor de riesgo desencadenante ya reportado en el metaanálisis de Dentali, donde describe un incremento del riesgo de trombosis de senos venosos con el uso de anticonceptivos orales (OR 5.59; 95\% IC 3.95 a $7.91 ; \mathrm{P}<0.01$ ) y en presencia de la mutación del gen de la protrombina 20210A (OR de 9.27; IC $95 \% 5.85$ a 14.67 p 0.01).

En el análisis de trombosis de miembro superior nuestra serie identificó trombofilia en 19\% para miembro superiores, incidencia un poco menor comparada con los resultados del trabajo de Linnemann en el análisis del registro MAISTHRO donde reportan un 32\% (30).

En el análisis de tratamiento encontramos en la mayoría de casos la terapia con warfarina fue la más frecuente en $74 \%$ de acuerdo con los lineamientos de tratamiento propuestos para estas entidades $(4,7,14,22)$, se describió tiempo de tratamiento mayor de 12 meses en $85 \%$, la suspensión de la terapia se documentó en $26 \%$ en casos de trombofilias menores o sin trombofilia documentada.

Nuestra serie reportó una recurrencia de $4.1 \%$, se identificó síndrome antifosfolípidos en dos pacientes con territorios involucrados lecho esplácnico y miembro inferior, se consideró dado la irregularidad en el seguimiento de los pacientes por circunstancias ajenas a los objetivos del servicio, este resultado se debe interpretar con cautela y consideramos existe un sesgo de selección, se espera controlar este sesgo en una fase prospectiva del estudio. Se resalta que en algunos pacientes no se puede suspender anticoagulación a pesar de no documentar trombofilia por severidad del evento donde prima el criterio clínico. El análisis de la calidad de pruebas diagnósticas se dificultó ya que no todas las historias clínicas describen las característica del método empleado, por lo que se dio valor a la interpretación de la prueba por el hematólogo tratante y valores reportados en la historia clínica, se espera controlar este sesgo de selección e información en una fase prospectiva. El estudio de las trombosis en sitios inusuales sigue siendo un objetivo importante en la línea de investigación de trombosis y hemostasia del servicio de hematología del Hospital San José.

Se concluye que existe una frecuencia alta para trombofilia en pacientes con trombosis en sitio inusual, siendo el síndrome antifosfolípidos la más frecuente en nuestra serie. Se considera pertinente el estudio de trombofilia en esta situación clínica. En los casos de trombosis de lecho esplácnico se puede contemplar dentro del estudio de hipercoagulabilidad la solicitud de la mutación JAK 2 v617f apoyados en los resultados de series internacionales

\section{Conflictos de interés}

Los autores no declaran conflicto de interés.

\section{Agradecimientos}

Agradecimiento al personal de archivo de historias clínicas y al departamento de sistemas del Hospital San José.

Agradecimiento al Departamento de Investigación del Hospital San José.

\section{Referencias}

1. Lussana F, Dentali F, Ageno W, Kamphuisen PW. Venous thrombosis at unusual sites and the role of thrombophilia. Semin Thromb Hemost 2007; 33(6): 582-587.

2. Margaglione M, Brancaccio V, De LD et al. Inherited thrombophilic risk factors and venous thromboembolism: distinct role in peripheral deep venous thrombosis and pulmonary embolism. Chest 2000; 118(5): 1405-1411.

3. White RH. The epidemiology of venous thromboembolism. Circulation 2003; 107(23 Suppl 1): I4-I8.

4. Martinelli I, Battaglioli T, Bucciarelli P, Passamonti SM, Mannucci PM. Risk factors and recurrence rate of primary deep vein thrombosis of the upper extremities. Circulation 2004; 110(5): 566-570.

5. Martinelli I, De S, V. Rare thromboses of cerebral, splanchnic and upperextremity veins. A narrative review. Thromb Haemost 2010; 103(6): 1136-1144.

6. Mai C, Hunt D. Upper-extremity deep venous thrombosis: a review. Am J Med 2011; 124(5): 402-407.

7. Saposnik G, Barinagarrementeria F, Brown RD, Jr. et al. Diagnosis and management of cerebral venous thrombosis: a statement for healthcare professionals from the American Heart Association/American Stroke Association. Stroke 2011; 42(4): 1158-1192.

8. Donadini MP, Ageno W. Unusual site thrombosis. Semin Hematol 2011; 48(4): 264-270.

9. Gosk-Bierska I, Wysokinski W, Brown RD, Jr. et al. Cerebral venous sinus thrombosis: Incidence of venous thrombosis recurrence and survival. Neurology 2006; 67(5): 814-819.

10. Martinelli I, Franchini M, Mannucci PM. How I treat rare venous thromboses. Blood 2008; 112(13): 4818-4823.

11. Torres JD, Cardona H, Alvarez L et al. Inherited thrombophilia is associated with deep vein thrombosis in a Colombian population. Am J Hematol 2006; 81(12): 933-937.

12. Dentali F, Gianni M, Crowther MA, Ageno W. Natural history of cerebral vein thrombosis: a systematic review. Blood 2006; 108(4): 1129-1134.

13. Dentali F, Crowther M, Ageno W. Thrombophilic abnormalities, oral contraceptives, and risk of cerebral vein thrombosis: a meta-analysis. Blood 2006; 107(7): 2766-2773.

14. Ageno W, Dentali F, Squizzato A et al. Evidence and clinical judgment: Treatment of cerebral vein thrombosis. Thromb Haemost 2010; 103(6): 1109-1115.

15. Coutinho J, De Bruijn SF, deVeber G, Stam J. Anticoagulation for cerebral venous sinus thrombosis. Cochrane Database Syst Rev 2011; (8): CD002005. 
16. Coutinho JM, Stam J. How to treat cerebral venous and sinus thrombosis. $J$ Thromb Haemost 2010; 8(5): 877-883.

17. Darwish MS, Plessier A, Hernandez-Guerra M et al.Etiology, management, and outcome of the Budd-Chiari syndrome. Ann Intern Med 2009; 151(3): 167-175.

18. De S, V, Martinelli I. Abdominal thromboses of splanchnic, renal and ovarian veins. Best Pract Res Clin Haematol 2012; 25(3): 253-264.

19. Dentali F, Galli M, Gianni M, Ageno W. Inherited thrombophilic abnormalities and risk of portal vein thrombosis. a meta-analysis. Thromb Haemost 2008; 99(4): 675-682.

20. Dentali F,Ageno W, Witt D et al. Natural history of mesenteric venous thrombosis in patients treated with vitamin $\mathrm{K}$ antagonists: a multi-centre, retrospective cohort study. Thromb Haemost 2009; 102(3): 501-504.

21. Holst AG, Jensen G, Prescott E. Risk factors for venous thromboembolism: results from the Copenhagen City Heart Study. Circulation 2010; 121(17): 18961903.

22. Stam J, De Bruijn SF, deVeber G. Anticoagulation for cerebral sinus thrombosis. Cochrane Database Syst Rev 2002; (4): CD002005.

23. Margetic S. Diagnostic algorithm for thrombophilia screening. Clin Chem Lab Med 2010; 48 Suppl 1: S27-S39.
24. Middeldorp S. Evidence-based approach to thrombophilia testing. $J$ Thromb Thrombolysis 2011; 31(3): 275-281.

25. Miyakis S, Lockshin MD, Atsumi T et al. International consensus statement on an update of the classification criteria for definite antiphospholipid syndrome (APS). J Thromb Haemost 2006; 4(2): 295-306.

26. James AH, Bushnell CD, Jamison MG, Myers ER. Incidence and risk factors for stroke in pregnancy and the puerperium. Obstet Gynecol 2005; 106(3): 509-516.

27. Treadwell SD, Thanvi B, Robinson TG. Stroke in pregnancy and the puerperium. Postgrad Med J 2008; 84(991): 238-245.

28. Fiorini A, Chiusolo P, Rossi E et al. Absence of the JAK2 exon 12 mutations in patients with splanchnic venous thrombosis and without overt myeloproliferative neoplasms. Am J Hematol 2009; 84(2): 126-127.

29. Tondeur S, Boutruche S, Biron-Andreani C, Schved JF. Prevalence of the JAK2 V617F mutation associated with splanchnic vein thrombosis. A 10-year retrospective study. Thromb Haemost 2009; 101(4): 787-789.

30. Linnemann B, Meister F, Schwonberg J, Schindewolf M, Zgouras D, LindhoffLast E. Hereditary and acquired thrombophilia in patients with upper extremity deep-vein thrombosis. Results from the MAISTHRO registry. Thromb Haemost 2008; 100(3): 440-446. 